\title{
Early Neuropathy Related to Oxaliplatin Treatment in Advanced and Recurrent Colorectal Cancer
}

\author{
TOMOYUKI NAGATA, KEN-ICHIRO FUKUDA, MIZUKI TAMAI, AKIHIRO TANIGUCHI, \\ HAJIME KAMIYA, KOSUKE KAMBE, YOSUKE KAMADA, GEORGE IWATA and NOBUKI YAMAOKA \\ Department of Surgery, Kyoto Chubu Medical Center, Kyoto, Japan
}

\begin{abstract}
Background/Aim: Chemotherapy dose adjustments in colorectal cancer are usually based on body surface area (BSA). The goal of this study was to investigate patients with nutritional disorder who developed early peripheral neuropathy due to inappropriate dose adjustment of oxaliplatin. Patients and Methods: The study subjects were 88 patients with advanced or recurrent colorectal cancer who underwent chemotherapy with oxaliplatin. The psoas muscle area (PMA) was used as a nutritional index. Mild (grades 0 1, MN group) and severe (grades 2-3, SN group) peripheral neuropathy was defined using neurotoxicity criteria of Debiopharm. Results: Severe peripheral neuropathy developed in 29 patients (33.0\%). The total oxaliplatin dose/PMA was significantly higher for the SN group $\left(107.6 \pm 8.5 \mathrm{mg} / \mathrm{cm}^{2}\right)$ and compared with the MN group $\left(53.8 \pm 6.0 \mathrm{mg} / \mathrm{cm}^{2}\right)$ in univariate $(p<0.0001)$ and multivariate $(p=0.012)$ analyses. Conclusion: In order to prevent peripheral neuropathy from chemotherapy for colorectal cancer, dose adjustment of oxaliplatin should be based on $P M A$, in addition to BSA.
\end{abstract}

Oxaliplatin is the key drug in standard chemotherapy regimens for the treatment of advanced and recurrent colorectal cancer. However, accumulated oxaliplatin induces peripheral neuropathy in some cases, and the chemotherapy regimen consequently has to be terminated. The current strategy in use of oxaliplatin is to make dose adjustments based on body surface area (BSA) (1), but we have encountered patients in whom the chemotherapy regimen could not be continued because of development of peripheral neuropathy earlier than expected.

Correspondence to: Tomoyuki Nagata, Ph.D., Department of Surgery, Kyoto Chubu Medical Center, 25 Yagiueno, Yagi-cho, Nantan-city, Kyoto 629-0197, Japan. Tel: +81 0771422510, Fax: +81 0771422096, e-mail: drgechum@koto.kpu-m.ac.jp

Key Words: Psoas muscle, chemotherapy, neuropathy, colorectal cancer.
Depletion of the psoas muscle has an adverse impact on gastrointestinal cancer treatment and early postoperative ambulation. We have shown that the psoas muscle volume assessed by computed tomography (CT) is related to the duration of chemotherapy and is an independent prognostic factor in patients with advanced colorectal cancer (2). In this study, we investigated the influence of the psoas muscle volume on dose adjustments of oxaliplatin in patients with advanced colorectal cancer.

\section{Patients and Methods}

Patient population and data collection. A total of 97 patients with advanced or recurrent colorectal cancer underwent chemotherapy with oxaliplatin at our Department between December 2009 and August 2017. Of these patients, five who underwent conversion to resection of metastatic lesions and four who were not followed-up were excluded from the study. The other patients were followed-up until discontinuation of oxaliplatin chemotherapy (January 31, 2018) by outpatient clinic consultation, and via telephone or letter. The median and mean follow-up periods were 102 and 130 days (range $=14-729$ days), respectively. A retrospective study of clinicopathological factors was performed. Approval for the study from our hospital Ethics Committee was waived because of the retrospective design.

Dose calculation and neuropathy grading. BSA was calculated by the Dubois formula as $0.007184 \times[\text { height }(\mathrm{m})]^{0.725} \times[$ weight $(\mathrm{kg})]^{0.425}$. The psoas muscle area (PMA) was calculated as the sum of the bilateral cross-sectional psoas muscle areas $\left(\mathrm{cm}^{2}\right)$ on CT. The total accumulated dose (TD) of oxaliplatin per unit BSA or PMA for each patient was defined as TD/BSA $\left(\mathrm{mg} / \mathrm{m}^{2}\right)$ and TD/PMA $\left(\mathrm{mg} / \mathrm{cm}^{2}\right)$, respectively. The severity of neuropathy was judged using the neurotoxicity criteria of Debiopharm (DEB-NTC) (Table I) (3).

Comparison of cases with mild and severe neuropathy. Associations between neuropathy severity and clinicopathological factors, including TD/BSA and TD/PMA, were examined in univariate and multivariate analyses of cases with mild (grades $0-1, \mathrm{MN}$ group) and severe (grades 2-3, SN group) neuropathy based on the DEBNTC criteria. Relationships with BSA and PMA were examined for each patient. Cut-off values of TD/BSA or TD/PMA were defined to discriminate between $\mathrm{MN}$ and $\mathrm{SN}$ groups using logistic regression analysis. The relationship between the actual dose 
Table I. Neurotoxicity criteria of Debiopharm (3).

Grade $0 \quad$ Nothing abnormal detected

Grade 1 Development of peripheral neurological symptoms Disappear within less than 7 days

Grade 2 Peripheral neurological symptoms persist for 7 or more days Without functional disorder

Grade 3 Development of functional disorder

administered to each patient in the SN group and the cut-off values were analyzed. In order to compare the number of cycles permitted in view of calculated doses from BSA and PMA in the SN group, the number of cycles of oxaliplatin administration before reaching each cut-off value was also examined.

Image analysis. CT scans for planning of staging were used for measuring the cross-sectional area of the psoas muscles at the inferior border of the third lumbar vertebra (L3) (Figure 1). PMA is normally assessed by measuring the psoas muscle at L3 because skeletal muscle and adipose tissue areas at L3 are significantly related to whole-body tissue mass in healthy adults (4) and patients with cancer (5). The muscle cross-sectional area was computed by summing tissue pixels and multiplying by the pixel surface area.

Statistical analysis. Statistical analysis and graphing were performed with JMP 7.0.1 (SAS Institute Inc., Tokyo, Japan). Continuous data are expressed as the median or mean, depending on the data distribution. A chi-square test and $t$-test (or Fisher test) were used for univariate comparisons. Multivariate analysis of independent factors related to severity of neuropathy was performed using a logistic regression model with variables that were significant factors in univariate analysis. In all analyses, a value of $p<0.05$ was considered to indicate significance.

\section{Results}

Severe neurotoxic adverse events developed in 29 patients, including 26 of grade 2 of the DEB-NTC classification, and three of grade 3. The PMA range was $2.65-22.07 \mathrm{~cm}^{2}$ and the mean PMA was $12.38 \pm 4.53 \mathrm{~cm}^{2}$. The chemotherapy regimens were oxaliplatin/5-fluorouracil/leucovorin (FOLFOX) $(\mathrm{n}=54)$ and capecitabine/oxaliplatin (CapeOX) $(n=34)$, with or without a molecular targeted drug for each regimen. No patient received neoadjuvant chemotherapy.

The clinicopathological features of patients of the SN and MN groups are listed in Table II. There were significant differences between groups: additional molecular targeted medicine was given more frequently to the $\mathrm{SN}$ group $(96.6 \%$ vs. $71.2 \%, p=0.0047$ ), body weight loss of more than $2 \mathrm{~kg}$ was more frequent in the SN group $(48.3 \%$ vs. $24.5 \%, p=0.0481)$, and both TD/BSA (588.9 \pm 43.5 vs. $445.9 \pm 30.5 \mathrm{mg} / \mathrm{m}^{2}$, $p=0.0085)$ and TD/PMA (107.6 $\pm 8.5 v s .53 .8 \pm 6.0 \mathrm{mg} / \mathrm{cm}^{2}$, $p<0.0001)$ were significantly higher in the $\mathrm{SN}$ group but there were no significant differences in factors associated with

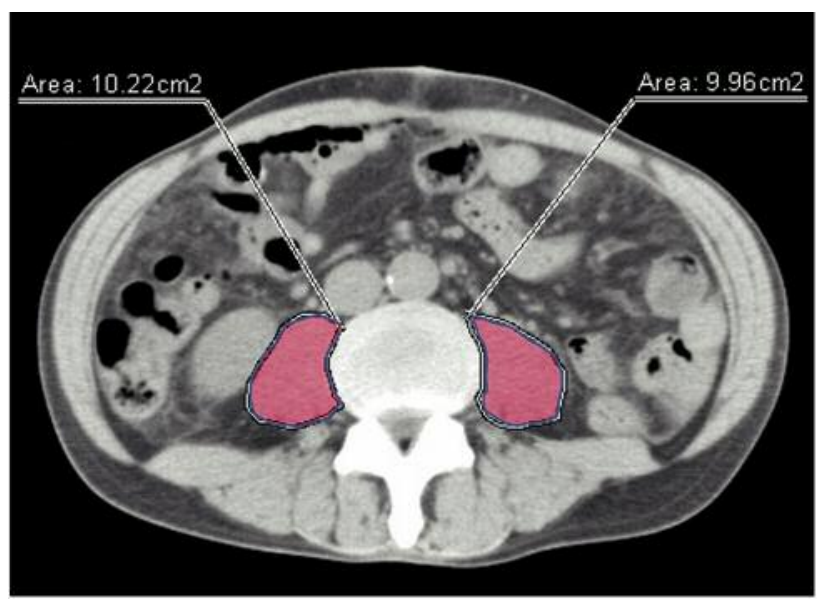

Figure 1. Total bilateral psoas muscle and all trunk cross-sectional areas in computed tomographic images at the inferior border of the third lumbar vertebra.

tumor histology or operative procedures. In multivariate analysis, body weight loss and TD/PMA were independent factors associated with severity of peripheral neuropathy.

Comparative analyses of BSA and PMA for all patients are shown in Figure 2. As shown graphically, for any given BSA, the PMA for patients of the SN group is distributed at a significantly lower range than that for the MN group. The optimal cut-off for TD/PMA was found to be $65.36 \mathrm{mg} / \mathrm{cm}^{2}$, with a sensitivity of $74.1 \%$, specificity of $71.9 \%$, and accuracy of $72.6 \%$ for prediction of peripheral neuropathy. An analysis using the actual dose showed that 20 patients were administered an oxaliplatin dose above the TD/PMA cut-off value. Among these patients, three received oxaliplatin at a total dose under the cut-off value for TD/BSA, but over the value for TD/PMA (Figure 3), and all three developed grade 2 neuropathy, but still underwent the next cycle of chemotherapy. An analysis of the permitted number of oxaliplatin cycles for the SN group revealed that for 13 patients, the administered dose did not reach the cut-off values of TD/BSA or TD/PMA. Among the other 16 patients, for 13 the TD/PMA cut-off was reached sooner than the TD/BSA cut-off. The difference in frequencies for reaching the two cutoffs ranged from 1 to 5, with an average of 2 (Figure 4).

\section{Discussion}

Oxaliplatin is a third-generation platinum drug that is particularly effective for treatment of gastrointestinal cancer and is included in the FOLFOX and XELOX regimens for advanced and recurrent colorectal cancer. Chemotherapyinduced peripheral neurotoxicity (CIPN) is one of the adverse effects of therapy with oxaliplatin. CIPN is an age-old problem but there are still many aspects of its mechanism that are 


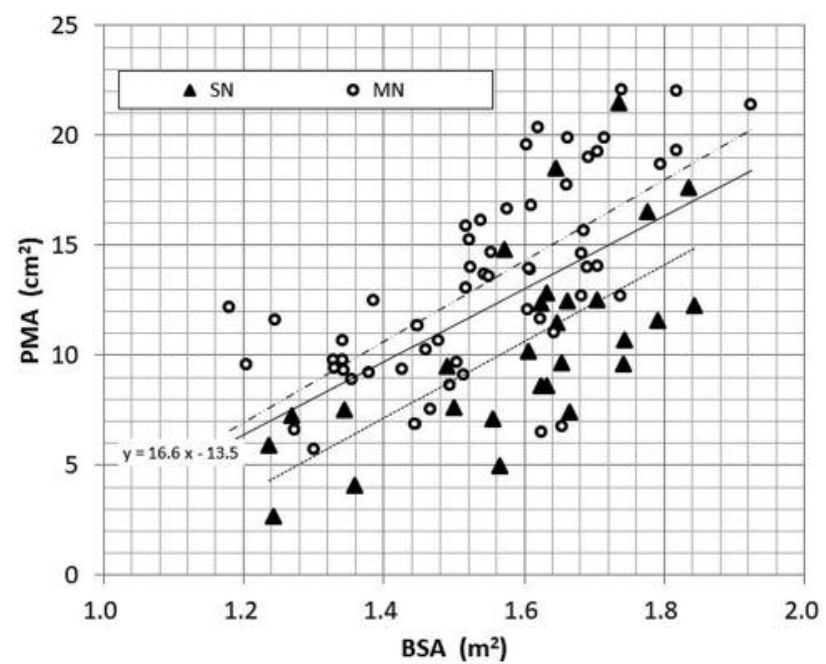

Figure 2. Relationship of body surface area (BSA) with psoas muscle area (PMA) for all patients with mild (MN) or severe (SN) neuropathy.

unclear and prevention has not been established. Several trials of drugs to control CIPN have not resulted in sufficient efficacy (6). CIPN is a complicated adverse event that may stop patients from continuing chemotherapy and worsens prognosis. CIPN is classified into axonopathy, neuronopathy and myelinopathy based on the etiological mechanism. Oxaliplatin causes neuronopathy, which occurs due to cell death in neural ganglia of the dorsal spinal nerve root. Neuronopathy is thought to be hard to reverse because of damage to the neuron itself, even if drug administration is stopped, but $40 \%$ of patients with neurotoxic adverse events show complete recovery 8 months after oxaliplatin is stopped (7).

Prevention and management of CIPN are likely to improve the results of cancer therapy and quality of life (QOL) of patients, but the main strategy at present is discontinuation of the offending drug. CIPN is thought to be related to the accumulated dose, and some approaches to prevention of symptoms have been developed (8). In the GERCOR study, the safety and efficacy of interruption of oxaliplatin after six cycles in a FOLFOX regimen was verified (9). As a result of this study, the Stop and Go strategy has become a standard approach in chemotherapy for advanced colorectal cancer. The limit for the cumulative dose of oxaliplatin is $680 \mathrm{mg} / \mathrm{m}^{2}$, with $30 \%$ of patients at risk of grade 2 peripheral neuropathy if this limit is exceeded (10). The limit for the accumulated dose in each case is calculated using BSA. However, some patients develop neuropathy sooner than predicted, and our experience suggests that prevention of neuropathy using this method may not always be adequate in clinical practice.

Depletion of skeletal muscle volume, characterized by increased decomposition and reduced synthesis of proteins in skeletal muscle tissue, results in marked depletion of lean body

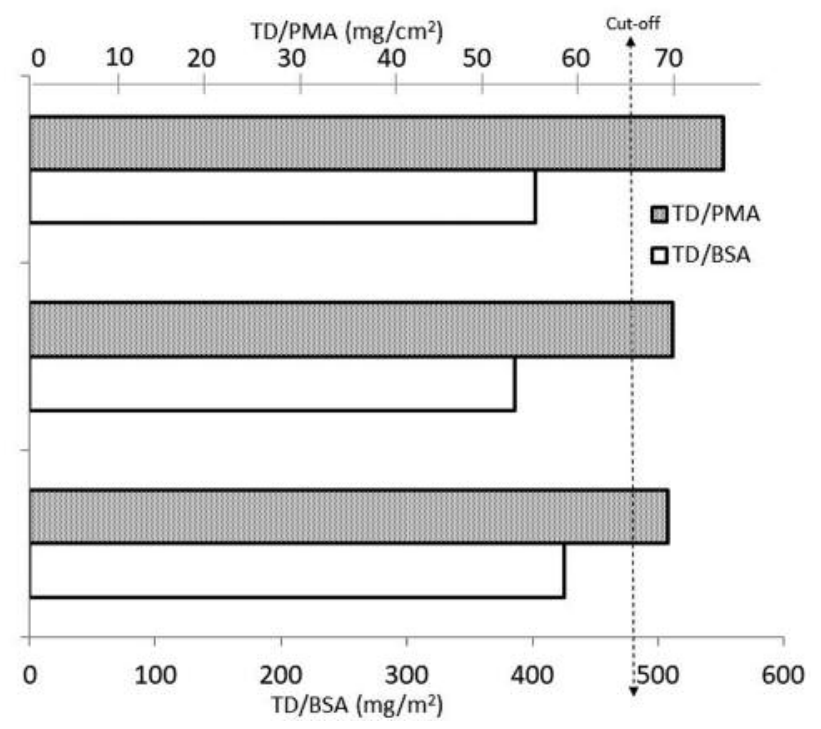

Figure 3. Three patients in the group with severe neuropathy received oxaliplatin at a total dose (TD) under the cut-off value for TD/body surface area (BSA), but over the value for TD/psoas muscle area (PMA).

mass (11). We have shown that the psoas muscle status on CT is related to the duration of chemotherapy and is an independent prognostic factor in advanced colorectal cancer (2). This muscle status may also be a useful index of physiological potential for prediction of the risk of complications after digestive surgery. Otsuji et al. found that skeletal muscle depletion increased morbidity, including liver failure, after major hepatectomy with extrahepatic bile duct resection (12), and Joglekar et al. showed that reduced muscle volume is a significant predictor of complications after pancreatectomy (13). We also suggested that the balance of psoas muscle volume and body trunk, named the 'PandA Ratio', might be a predictor of complications after laparoscopyassisted gastrectomy in patients with gastric cancer (14).

Based on these findings, reduction of the psoas muscle volume is now widely used as an index for poor outcomes of cancer treatment. Therefore, we analyzed the impact of evaluation of PMA on CT images on peripheral neuropathy induced by oxaliplatin chemotherapy in patients with advanced and recurrent colorectal cancer. BSA is generally calculated from the patient's height and weight, but body weight might be modified if the patient develops edema or stored ascites because of inflammation or malnutrition caused by the original disease. Consequently, BSA may vary markedly. CT images are utilized for cancer staging and planning of therapy, and are convenient for evaluation of tissue volume such as skeletal muscle. These images are routinely available in clinical records and this assessment places no additional stress on the patient. 
Table II. The clinicopathological features of patients of the severe neuropathy (SN) and mild neuropathy (MN) groups.

\begin{tabular}{|c|c|c|c|c|c|c|}
\hline & & \multicolumn{3}{|c|}{ Univariate analysis } & \multicolumn{2}{|c|}{ Multivariate analysis } \\
\hline & & $\mathrm{SN}$ group & MN group & $p$-Value & $95 \% \mathrm{CI}$ & $p$-Value \\
\hline Number & & 29 & 59 & & & \\
\hline \multicolumn{7}{|l|}{ Background } \\
\hline Age, years & Mean \pm SD & $66.3 \pm 2.0$ & $68.9 \pm 1.4$ & 0.283 & & \\
\hline Gender, n & Female/male & $14 / 15$ & $24 / 35$ & 0.648 & & \\
\hline $\mathrm{BSA}, \mathrm{m}^{2}$ & Mean \pm SD & $1.59 \pm 0.03$ & $1.55 \pm 0.02$ & 0.253 & & \\
\hline $\mathrm{BW}, \mathrm{kg}$ & Mean \pm SD & $57.7 \pm 1.8$ & $55.2 \pm 1.3$ & 0.264 & & \\
\hline Status, $\mathrm{n}$ & Stage4/rec. & $19 / 10$ & $35 / 24$ & 0.646 & & \\
\hline \multicolumn{7}{|l|}{ Pathological factors } \\
\hline Origin, $\mathrm{n}$ & Colon/rectum & $19 / 10$ & $37 / 22$ & $>0.99$ & & \\
\hline Tumor size, $\mathrm{mm}$ & Mean \pm SD & $52.1 \pm 4.9$ & $56.8 \pm 3.4$ & 0.428 & & \\
\hline $\mathrm{pT}, \mathrm{n}$ & $1-4 a / 4 b$ & $23 / 3$ & $52 / 5$ & 0.701 & & \\
\hline $\mathrm{pN}, \mathrm{n}$ & $0 / 1-3$ & $6 / 21$ & $13 / 42$ & $>0.99$ & & \\
\hline Macroscopic type, $n$ & Diffuse/focal & $7 / 18$ & $20 / 37$ & 0.615 & & \\
\hline Histology, $\mathrm{n}$ & Well/other & $11 / 16$ & $23 / 36$ & $>0.99$ & & \\
\hline pStage, $n$ & 0 -II/III, IV & $4 / 24$ & $10 / 48$ & $>0.99$ & & \\
\hline ly, n & $0 / 1-3$ & $1 / 24$ & $5 / 51$ & 0.661 & & \\
\hline $\mathrm{v}, \mathrm{n}$ & $0 / 1-3$ & $11 / 14$ & $21 / 35$ & 0.628 & & \\
\hline \multicolumn{7}{|l|}{ Operative factors } \\
\hline Approach, n & Laparoscopy/open & $8 / 18$ & $18 / 39$ & $>0.99$ & & \\
\hline Original lesion, $\mathrm{n}$ & Removed/remnant & $25 / 2$ & $54 / 2$ & 0.593 & & \\
\hline LND, $\mathrm{n}$ & D1-2/D3 & $12 / 14$ & $25 / 30$ & $>0.99$ & & \\
\hline Operative time, $\min$ & Mean \pm SD & $266.0 \pm 33.2$ & $282.4 \pm 22.4$ & 0.683 & & \\
\hline Blood loss, g & & $377.0 \pm 214.2$ & $601.0 \pm 144.7$ & 0.389 & & \\
\hline \multicolumn{7}{|l|}{ Chemotherapy factors } \\
\hline Regimen, $\mathrm{n}$ & FOLFOX/CapeOX & $17 / 12$ & $37 / 22$ & 0.817 & & \\
\hline Molecular targeted therapy, $\mathrm{n}$ & Yes/no & $28 / 1$ & $42 / 17$ & 0.005 & $-2.599--0.168$ & 0.053 \\
\hline No. of cycles & Mean \pm SD & $6.6 \pm 0.5$ & $5.7 \pm 0.4$ & 0.156 & & \\
\hline$\Delta \mathrm{BW}, \mathrm{kg}$ & $>-2.0 / \leq-2.0$ & $15 / 14$ & $40 / 13$ & 0.048 & $-1.307--0.094$ & 0.027 \\
\hline Total OX. dose/BSA, mg/m² & Mean \pm SD & $588.9 \pm 43.5$ & $445 \pm 30.5$ & 0.009 & $-0.627-0.708$ & 0.863 \\
\hline Total OX. dose/PMA, mg/cm² & Mean \pm SD & $107.6 \pm 8.5$ & $53.8 \pm 6.0$ & $<0.000 *$ & $0.224-1.626$ & 0.012 \\
\hline
\end{tabular}

BSA: Body surface area; BW: body weight; FOLFOX: fluorouracil/leucovorin/oxaliplatin; CapeOX: capecitabine/oxaliplatin; LND: lymph node dissection; ly: histological lymph system invasion; MN: mild neuropathy; pN: pathological N grade; SN: severe neuropathy; pT: pathological T grade; v: histological vessel invasion; rec.: recurrence; Well: well-differentiated. Significant differences are shown in bold.

Several molecular targeted drugs have emerged as breakthroughs in advanced and recurrent colorectal cancer therapy with cytotoxic drugs (15). In univariate analysis in the present study, there was a significant difference of neuropathy grade with and without use of these drugs. In particular, 28 out of 29 patients with severe neuropathy had also received molecular targeted drugs: 27 received an antibody to vascular endothelial growth factor (VGEF) and one was treated with an anti-epidermal growth factor receptor (EGFR) drug. In advanced and recurrent colorectal cancer, mutation tests for RAS gene are widely used to select patients for treatment with anti-VEGF or anti-EGFR. In previous reports, patients with colorectal cancer who received anti-VEGF drugs had adverse events of oral mucosal ulcers and diarrhea (16), but neurotoxic adverse events in molecular targeted therapy have not been described. In the current study, molecular targeted therapy was not an independent risk factor in multivariate analysis. We interpret this result as indicating that the worsened neurotoxicity was due to another factor related to nutritional disorder caused by the digestive adverse effects mentioned above.

Body weight loss could be a factor that increases the incidence of various adverse events and causes reduced compliance with chemotherapy after surgery. In a retrospective multivariate analysis of 103 patients with gastric cancer, Aoyama et al. found that body weight loss of $15 \%$ or more at 1 month after surgery was a significant risk factor for discontinuation of S-1 adjuvant chemotherapy (17). Yamaoka et al. showed that body weight loss during chemotherapy was derived from depletion of skeletal muscle volume (18), and in the current study we found that body weight loss of $2 \mathrm{~kg}$ or more was an independent risk factor for severe neuropathy. These finding suggest that malnutrition with depletion of 


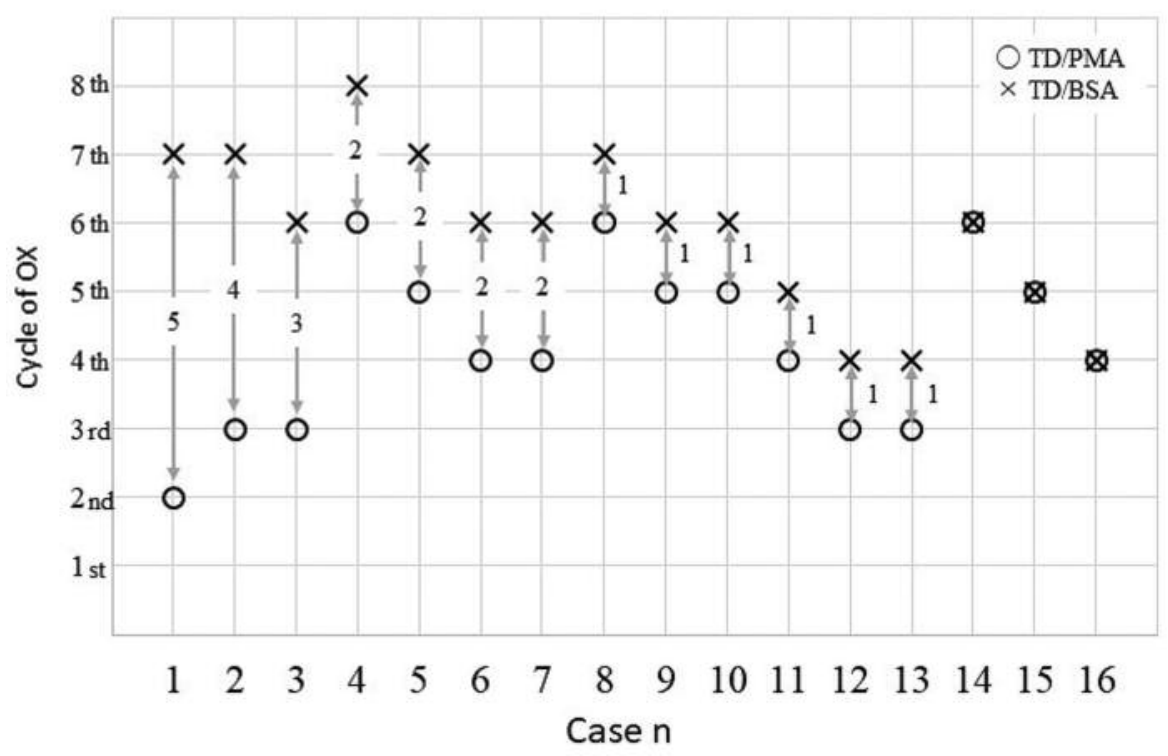

Figure 4. The difference in number of cycles required to reach the two cut-offs for oxaliplatin $(O X)$ in the group with severe neuropathy. TD/BSA: Total dose by body surface area; TD/PMA: total dose by psoas muscle area.

skeletal muscle can have a significant impact on the mechanism of development of neuropathy.

Reduction of skeletal muscle is often considered to be involved in weight loss, but skeletal muscle volume reduction itself is not often used as an index for drug dose control. The skeletal muscle volumes of patients with the same BSAs are not necessarily equal, but in actual clinical practice, dose adjustments of oxaliplatin are calculated from the BSA. Therefore, the same dose of drugs might be administered to patients with different muscle volumes. Our analysis of the relationship between muscle volume and BSA showed that patients with severe neuropathy had smaller volumes of psoas muscle than those with mild neuropathy, despite BSAs being equal. The approximate linear relationship between BSA and PMA is: $P M A=B S A \times 16.6-13.5$. This equation indicates that PMAs of $10,11.5$ and $13 \mathrm{~cm}^{2}$ are equivalent to approximate BSAs of $1.4,1.5$ and $1.6 \mathrm{~m}^{2}$, respectively.

An increase in the concentration of intracellular glutathione (GSH) has been shown to have a strong correlation with resistance to cisplatin therapy in leukemia and ovarian cancer cells $(19,20)$. This finding has focused attention on the role of GSH in the mechanism of resistance to cisplatin therapy. Nishimura et al. found that increased immunohistological activity of glutathione S-transferase (GST), which mediates the GSH conjugation reaction with cisplatin, significantly reducing the anticancer efficacy of cisplatin chemotherapy (21). Therefore, conjugation to GSH is considered to be an important factor in platinum-based chemotherapy. GSH is a tri-peptide that is synthesized in the liver and has antioxidant properties, including elimination of reactive oxygen species that develop inside the cell. GSH decreases in skeletal muscle and increases in liver tissue during exercise (22), and GSH secreted into blood is taken up by skeletal muscle. This results in removal of oxygen radicals generated by exercise and prevents fatigue. Muscle tissue does not include a large amount of GSH itself, but oxidized glutathione (GSSG) increases and GSH decreases in atrophied muscle due to stress or cast immobilization (23). These reports suggest that patients with low PMA also have reduced metabolism and detoxification activities due to lower levels of GSH.

Dose planning using PMA may be effective for more precise prevention of neuropathy compared to planning with BSA alone. In the present study, we found that a small population of patients should have stopped chemotherapy with oxaliplatin one or two cycles earlier. For example, patients with a smaller PMA might receive maintenance chemotherapy sooner than the commonly suggested time. However, careful planning is required to stop oxaliplatin sooner than suggested, since this drug has a high response rate in colorectal cancer. Therefore, there is a need to determine the point at which there will be no reduction of anticancer efficacy in more cases, as performed in the IDEA (24) and OPTIMOX (8) trials. However, stopping a drug suspected to be causing CIPN almost always reduces the anticancer effect, except for a few reports in colorectal cancer. Therefore, patients with severe CIPN should be treated with an alternative drug when possible. If no alternative drugs are available, stopping or 
reducing therapy requires careful consideration and informed consent, based on the patient understanding the advantages and disadvantages. Clear planning is required before reducing or stopping a suspect drug in adjuvant therapy with a fixed and limited schedule, or in curative chemotherapy for hematological cancer. Patients should have the chance for full recovery and prevention of a decline in quality of life by completing these therapeutic courses.

In conclusion, improved prevention of peripheral neuropathy in chemotherapy for colorectal cancer may be achieved by dose adjustment of oxaliplatin based on PMA, in addition to BSA.

\section{Conflicts of Interest}

The Authors declare no conflicts of interest associated with this article.

\section{Authors' Contributions}

Tomoyuki Nagata designed the study, wrote the initial draft of the article and contributed to analysis and interpretation of data. All other Authors contributed to data collection and interpretation, and critically reviewed the article. All Authors approved the final version of the article, and agree to be accountable for all aspects of the work in ensuring that questions related to the accuracy or integrity of any part of the work are appropriately investigated and resolved.

\section{References}

1 Puisset F, Schmitt A and Chatelut E: Standardization of chemotherapy and individual dosing of platinum compounds. Anticancer Res 34: 465-470, 2014. PMID: 24403502.

2 Nagata T, Nakase Y, Nakamura K, Sougawa A, Mochiduki S, Kitai S and Inaba S: Prognostic impact of a nutritional index including muscle volume in stage 4 colorectal cancer. In Vivo 30: 885-892, 2016. PMID: 27815476. DOI: 10.21873 /invivo.11009.

3 Nishioka M1, Shimada M, Kurita N, Iwata T, Morimoto S, Yoshikawa K, Higashijima J, Miyatani T and Kono T: The Kampo medicine, Goshajinkigan, prevents neuropathy in patients treated by FOLFOX regimen. Int J Clin Oncol 16(4): 322-327, 2011. DOI: 10.1007/s10147-010-0183-1.

4 Shen W, Punyanitya M, Wang Z, Gallagher D, St-Onge MP, Albu J, Heymsfield SB and Heshka S: Total body skeletal muscle and adipose tissue volumes: Estimation from a single abdominal cross-sectional image. J Appl Physiol 97: 2333-2338, 2004. PMID: 15310748. DOI: 10.1152/japplphysiol.00744.2004.

5 Mourtzakis M, Prado CM, Lieffers JR, Reiman T, McCargar LJ and Baracos VE: A practical and precise approach to quantification of body composition in cancer patients using computed tomography images acquired during routine care. Appl Physiol Nutr Metab 33: 997-1006, 2008. PMID: 18923576. DOI: 10.1139/H08-075.

6 Hirayama Y, Sasaki J, Dosaka-Akita H and Isitani L: Survey of the management of chemotherapy-induced peripheral neuropathy in Japan: Japanese Society of Medical Oncology. ESMO Open $l$ : e000053, 2016. PMID: 27843610. DOI: 10.1136/esmoopen2016-000053.

7 Argyriou AA, Bruna J, Marmiroli $\mathrm{P}$ and Cavaletti G: Chemotherapy-induced peripheral neurotoxicity (CIPN): An update. Crit Rev Oncol Hematol 82: 51-77, 2012. PMID: 21908200. DOI: 10.1016/j.critrevonc.2011.04.012.

8 Otake A, Yoshino K, Ueda Y, Sawada K, Mabuchi S, Kimura T, Kobayashi E, Isobe A, Egawa-Takata T, Matsuzaki S, Fujita M and Kimura T: Usefulness of duloxetine for paclitaxel-induced peripheral neuropathy treatment in gynecological cancer patients. Anticancer Res 35: 359-364, 2015. PMID: 25550572.

9 Tournigand C, André T, Achille E, Lledo G, Flesh M, MeryMignard D, Quinaux E, Couteau C, Buyse M, Ganem G, Landi B, Colin P, Louvet C and de Gramont A: FOLFIRI followed by FOLFOX6 or the reverse sequence in advanced colorectal cancer: A randomized GERCOR study. J Clin Oncol 22: 229337, 2004. PMID: 14657227. DOI: 10.1200/JCO.2004.05.113.

10 Kono T, Mishima H, Shimada M, Morita S, Sakamoto J and GONE Investigators: Preventive effect of goshajinkigan on peripheral neurotoxicity of FOLFOX therapy: A placebocontrolled double-blind randomized phase II study (the GONE Study). Jpn J Clin Oncol 39: 847-849, 2009. PMID: 19734172. DOI: $10.1093 /$ jjco/hyp100.

11 Kotler DP: Cachexia. Ann Intern Med 133: 622-634, 2000. PMID: 11033592.

12 Otsuji H, Yokoyama Y, Ebata T, Igami T, Sugawara G, Mizuno $\mathrm{T}$ and Nagino M: Preoperative sarcopenia negatively impacts postoperative outcomes following major hepatectomy with extrahepatic bile duct resection. World J Surg 39: 1494-1500, 2015. PMID: 25651963. DOI: 10.1007/s00268-015-2988-6.

13 Joglekar S, Asghar A, Mott SL, Johnson BE, Button AM, Clark E and Mezhir JJ: Sarcopenia is an independent predictor of complications following pancreatectomy for adenocarcinoma. J Surg Oncol 111: 771-775, 2015. PMID: 25556324. DOI: 10.1002/jso.23862.

14 Nagata T, Nakase Y, Nakamura K, Sougawa A, Mochiduki S, Kitai S and Inaba S: Impact of nutritional status on outcomes in laparoscopy-assisted gastrectomy. J Surg Res 219: 78-85, 2017. PMID: 29078914. DOI: 10.1016/j.jss.2017.05.118.

15 Moriarity A, O'Sullivan J, Kennedy J, Mehigan B and McCormick P: Current targeted therapies in the treatment of advanced colorectal cancer: A review. Ther Adv Med Oncol 8: 276-293, 2016. PMID: 27482287. DOI: 10.1177/17588340 16646734 .

16 Tol J, Cats A, Mol L, Koopman M, Koopman M, Bos MM, van der Hoeven JJ, Antonini NF, van Krieken JH and Punt CJ: Gastrointestinal ulceration as a possible side-effect of bevacizumab which may herald perforation. Invest New Drugs 26: 393-397, 2008. PMID: 18335169. DOI: 10.1007/s10637008-9125-4.

17 Aoyama T, Yoshikawa T, Shirai J, Hayashi T, Yamada T, Tsuchida K, Hasegawa S, Cho H, Yukawa N, Oshima T, Rino Y, Masuda $\mathrm{M}$ and Tsuburaya A: Body weight loss after surgery is an independent risk factor for continuation of S-1 adjuvant chemotherapy for gastric cancer. Ann Surg Oncol 20: 2000-2006, 2013. PMID: 23242818. DOI: 10.1245/s10434-012-2776-6.

18 Yamaoka Y, Fujitani K, Tsujinaka T, Yamamoto K, Hirao M and Sekimoto M: Skeletal muscle loss after total gastrectomy, exacerbated by adjuvant chemotherapy. Gastric Cancer 18: 382389, 2015. PMID: 24715254. DOI: 10.1007/s10120-014-0365-z. 
19 Godwin AK, Meister A, O'Dwyer PJ, Huang CS, Hamilton TC and Anderson ME: High resistance to cisplatin in human ovarian cancer cell lines is associated with marked increase of glutathione synthesis. Proc Natl Acad Sci 89: 3070-3074, 1992. PMID: 1348364.

20 Andrews PA: Mechanisms of acquired resistance to cisplatin. Cancer Treat Rep 66: 471-474, 1982.

21 Nishimura T, Newkirk K, Sessions RB, Andrews PA, Trock BJ, Rasmussen AA, Montgomery EA, Bischoff EK and Cullen KJ: Immunohistochemical staining for glutathione S-transferase predicts response to platinum-based chemotherapy in head and neck cancer. Clin Cancer Res 2: 1859-1865, 1986. PMID: 9816141.

22 Sen CK, Marin E, Kretzschmar M and Hänninen O: Skeletal muscle and liver glutathione homeostasis in response to training, exercise, and immobilization. J Appl Physiol 73: 1265-1272, 1992. PMID: 1360001. DOI: 10.1152/jappl.1992.73.4.1265.

23 Kondo H, Miura M, Kodama J, Ahmed SM and Itokawa Y: Role of iron in oxidative stress in skeletal muscle atrophied by immobilization. Pflugers Arch 421: 295-297, 1992. PMID: 1528723.
24 André T, Vernerey D, Mineur L, Bennouna J, Desrame J, Faroux R, Fratte S, Hug de Larauze M, Paget-Bailly S, Chibaudel B, Bez J, Dauba J, Louvet C, Lepere C, Dupuis O, Becouarn Y, Mabro M, Egreteau J, Bouche O, Deplanque G, Ychou M, Galais MP, Ghiringhelli F, Dourthe LM, Bachet JB, Khalil A, Bonnetain F, de Gramont A and Taieb J: Three versus 6 months of oxaliplatin-based adjuvant chemotherapy for patients with stage III colon cancer: Disease-free survival results from a randomized, open-label, international duration evaluation of adjuvant (IDEA) France, phase III trial. J Clin Oncol 36: 14691477, 2018. PMID: 29620995. DOI: 10.1200/JCO.2017.76.0355.

Received January 22, 2019

Revised February 6, 2019

Accepted February 7, 2019 